\title{
EDITORIAL
}

\section{Expressed emotion: current status ${ }^{1}$}

Almost three decades of controversy have surrounded the concept of 'expressed emotion'. This fascinating saga has been carefully chronicled in the recent book by Leff and Vaughr, Expressed Emotion in Families (1985). The original work derived from the unexpected finding that long-stay patients survived longer in community settings and maintained better social functioning when they were discharged to lodgings, siblings, or distant relatives, than when they returned to parental or spousal households, or to professionally staffed hostels (Brown et al. 1958). The emotional response of key household members to the patient's behavioural disturbance appeared to be the best predictor of successful transfer to the community.

Criticism of the crude methodology employed in this study and a second study (Brown et al. 1962) led to further replications (Brown et al. 1972; Vaughn \& Leff, 1976a) which employed standardized interview and rating techniques, the Camberwell Family Interview (Brown \& Rutter, 1966; Vaughn \& Leff, 1976b). These studies both replicated the earlier findings that an index of the emotional response of household members to patients' disturbed behaviour during the three months prior to a hospital admission for an episode of florid schizophrenia was the best single predictor of the recurrence of florid episodes in the 9 months after discharge back to the same household. The predictive power of the 'expressed emotion' index remained intact when prognostic variables such as symptom patterns, work impairment, severity of behavioural disturbance, duration of illness, age and pre-morbid social adjustment were partialled out in a series of contingency analyses. Although past impairment of work function and disturbed behaviour were strongly associated with both relapse and high 'expressed emotion' (EE), these associations largely disappeared when examined in conjunction with the relatives' emotional responses. It was concluded, therefore, that the association between relapse and disturbed behaviour was mediated through the high EE response of household members to that behaviour and not through the behaviour itself.

The care taken in these replication studies is exemplary. But before drawing definitive conclusions which link the emotional responses of household members to the course of schizophrenia a number of issues must be considered. These include: (i) What factors provoke high EE responses? (ii) What is the nature of the association between high EE responses and recurrence of florid episodes of schizophrenia? (iii) What factors moderate the negative predictive power of the EE index? (iv) Is EE a specific predictor of schizophrenic phenomenology?

\section{FACTORS PROVOKING HIGH EE RESPONSES}

The high EE responses that have proved consistent predictors of a relapsing course for schizophrenia have usually concerned the patient's behaviour at a time of major social crisis preceding a hospital admission. In these circumstances the factors that may provoke highly critical or over-involved responses in relatives are likely to be complex. Brown et al. (1962) postulated that the high emotional responses of relatives derived in part from a response to disturbed past or current behaviour of the patient, in part from the personality of the relative, and in part from an interaction between attitudes toward the patient and the developing illness, especially where deteriorating social performance was prominent. Vaughn (1977) studied the content of relatives' critical responses and observed that the stimuli for highly critical responses were predominantly long standing interpersonal deficits rather than florid symptoms and behaviour disturbance which could be more clearly considered features of the illness. Miklowitz et al. (1983) found a similar association between emotional over-involvement

\footnotetext{
' Address for correspondence: Dr I. R. H. Falloon, Buckingham Mental Health Service, Buckingham Hospital, Buckıngham MK18 INU.
} 
(EOI) and poor pre-morbid social competence. Because the florid symptoms of schizophrenia are essentially cognitive phenomena and are not linked in a highly specific way to overt behaviour disturbance, it is hardly surprising that they have not been found to provoke specific patterns of response in caregivers. However, there is further evidence to suggest that certain patterns of overt behavioural disturbance are more burdensome for relatives than others. Unpredictable threatening behaviour has been consistently reported as most difficult for relatives to tolerate (Grad \& Sainsbury, 1963; Creer \& Wing, 1974; Greenley, 1979; Hatfield, 1979; Hoenig, 1974; Test \& Stein, 1980). The deficit or 'negative' symptoms of schizophrenia appear more likely to provoke critical responses when relatives do not attribute them to an illness process (Vaughn \& Leff, 1981). Thus, there appears to be an association between the patient's past and current patterns of behavioural disturbance and high EE responses, but further careful research is required to fully elucidate its nature.

The suggestion that the personality of the relative may be a factor in determining their emotional responsiveness to florid schizophrenia has not been explored. The possibility that high 'expressed emotion' in relatives may be a behavioural marker of a genetic vulnerability factor has been suggested (Goldstein, 1985), but Brown's observation that high 'expressed emotion' represented the emotional responses of 'ordinary families' does not support a theory of family-based psychopathology (Brown, 1985). Furthermore, spouses are just as critical as parents, and high EE responses have been observed in the caregivers of patients with a wide variety of mental disorders.

High EE responses have been strongly associated with high levels of family burden (Brown et al. 1958). Although it is evident that during the pre-admission crisis the major source of that burden is likely to be the patient's disturbed behaviour, it is also probable that other concurrent stressors may limit the caregiver's ability to cope in an effective manner and lead to a greater negative emotional response towards the patient. Relatively little research has examined the interaction between emotional responses and the coping skills of family members, or stress factors other than the patient and his or her illness. Vaughn (1976) demonstrated a poorer course of schizophrenia where parental conflict pre-existed with high EE. Birchwood (1983) found that the coping strategies employed to deal with behavioural disturbance commonly found in schizophrenia appeared to be associated with the subsequent course of the condition. Miklowitz et al. (1984) showed that the critical and over-involved attitudes expressed in the Camberwell Family Interview were, indeed, markers of critical and intrusive behaviours in actual family problem solving discussions. It has been noted that the EE levels, especially criticism, peak during the patient's florid episode and decline once the florid symptoms and associated behaviour disturbance remit. In addition, expressed emotion levels tend to increase somewhat with chronicity (Brown et al. 1972; Birchwood 1983).

These data support the view that high expressed emotion levels are indicative of difficulties in coping with severe stress in the household, particularly that associated with the index patient and his behavioural difficulties. However, it cannot be assumed that low EE caregivers are effective at coping with all sources of environmental stress. Leff \& Vaughn (1980) noted an association between stressful life events and florid episodes in persons living in low EE households. This suggests that these families may provide an ineffective buffer against extrafamilial stressors. However, neuroleptic medication appears to have a prophylactic effect in reducing the risk of recurrence under these circumstances (Leff et al. 1983).

It is evident that understanding of the origins of 'expressed emotion' remains crude, but it is likely to reside in complex interactions between patient and caregivers.

\section{LINK BETWEEN EXPRESSED EMOTION AND THE COURSE OF SCHIZOPHRENIA}

High expressed emotion in at least one household member during the pre-admission crisis raises the probability of subsequent florid episodes of schizophrenic symptoms fourfold. Such a strong association has led researchers to search for evidence to link these two correlates in a causal hypothesis. It must be demonstrated that the presence of high expressed emotion responses contribute in a direct manner to the emergence of florid episodes of schizophrenic symptoms. Conversely, the absence of high expressed emotion responses should specifically inhibit the 
emergence of a florid episode of schizophrenia. Definitive experiments of this hypothesis have not been conducted. However, several attempts to establish mediating links between EE and schizophrenic symptomatology have been made.

Two studies based upon the theory that florid symptoms of schizophrenia increase with physiological arousal have suggested that the index patient's galvanic skin response (GSR) habituation is slower in the presence of a household member who has expressed high EE response than in the presence of a family member who has not displayed high EE characteristics toward the patient (Tarrier et al. 1979; Sturgeon et al. 1981). Arousal was not increased by the high. EE relative; instead, the low EE relative exerted a calming effect. Thus, the apparent benefits of living in a low EE household may be derived from the calming effects of family members who cope effectively with the behaviour disturbance of florid schizophrenia and promote a low stress home environment. However, all the predictive studies of EE have sampled only those patients who were initially admitted to hospital. This suggests that even many low EE households were unable to cope with the patient's behaviour disturbance at that time, particularly when the added stress of a recent life event was present (Leff \& Vaughn, 1980). It would be important to know whether those patients who were admitted because the family could no longer tolerate their disturbance generated more high EE responses than those admitted for predominantly medical management consideration.

Two studies have attempted to directly change high 'expressed emotion' or reduce its impact upon the clinical course of schizophrenia. Leff et al. (1982) concluded that a low rate of major exacerbations in patients who returned to live with relatives who had shown high EE responses in the pre-admission crisis but had low EE responses (mainly on the criticism variable) at 9-months follow-up provided evidence for a causal link between $E E$ and a relapsing course. Half the sample showed low EE responses after psychosocial interventions aimed at reducing high EE responses or their impact on the index patient. However, the rate of recurrence remained low in several cases with high EE at follow-up as well. In these cases a reduction in the amount of interpersonal contact with high EE persons was thought to account for the lowered risk of recurrence.

In contrast, a family intervention study by Hogarty et al. (1986) found continued high risk of recurrence where the EE index was high at follow-up even when interpersonal contact was low. However, where social skills training, designed to reduce interpersonal vulnerability, was added to family stress management, the recurrence rate became minimal, even in those households where EE remained high at follow-up. Although in this study follow-up EE ratings were not all based upon family member interviews, it is apparent that the high EE alone is an erratic predictor of the clinical course of schizophrenia. Furthermore, persons living in high contact with high EE relatives represent only one quarter of patients with schizophrenia who are resident in family settings. Thus, despite research evidence for the significance of the EE index as a prognostic factor, only a modest proportion of sufferers from schizophrenia are affected by this variable. However, perhaps the most optimistic feature of these intervention studies is that no patient living in a low EE household has experienced a florid episode of schizophrenia. The manner in which low EE promotes health in persons vulnerable to schizophrenic disorders would appear a potentially fruitful area for future study.

The causal hypothesis that links high EE households with an increased risk of florid schizophrenic episodes assumes that high EE responses remain constant over follow-up. Evidence does not fully support this assumption. The EE components are generally lower after recovery from an acute episode of schizophrenia, especially the criticism variable (Brown et al. 1972; Dulz \& Hand, 1986; Leff et al. 1982; Hogarty et al. 1986). This suggests that criticism is essentially a state measure not a stable trait, or as Brown et al. (1972) suggested, a 'latent' characteristic of households that is activated by the disturbed behaviour of the index patient. To date no study has conducted serial measures of EE to determine whether the high EE state of the household precedes the index patient's behaviour disturbance and contributes to a florid exacerbation, or whether behaviour disturbance triggers high EE responses in the household.

One family intervention study suggested that effective stress management and optimal drug prophylaxis may merely serve to reduce the social disruption and stress associated with recurrent 
episodes of schizophrenia, rather than modify the characteristic recurring nature of the disorder (Falloon et al. 1985). Prospective studies which enable the symptomatic state of patients to be reliably assessed on a week-to-week basis are needed to examine this issue.

It may be concluded that the association between EE and the course of schizophrenia is of an actuarial nature and that current understanding of the exact nature of its predictive function is limited. Current evidence that high EE responses in families cause or even contribute to the onset or exacerbation of schizophrenia should be viewed with caution. From a clinical standpoint, a positive approach to families that emphasizes the association between the ability of family members to cope efficiently with episodes of schizophrenia and the reduction in the frequency of subsequent major exacerbations is likely to enhance the cooperation of family caregivers as well as the development of effective psychosocial support programmes.

\section{FACTORS THAT MODERATE THE ASSOCIATION BETWEEN EE AND CLINICAL OUTCOME}

Two factors have been repeatedly examined for their impact upon the level of association between the EE index and clinical course of schizophrenia. The first concerns the amount of 'face-to-face' contact between the index patient and the person(s) expressing high EE. Brown et al. (1972) and Vaughn \& Leff (1976a) found a significantly lower proportion of cases who relapsed when the time in contact with high EE persons was less than 35 hours per week at the follow-up point. This effect was not replicated in the California Study (Vaughn et al. 1984), although a clear trend was observed. Two other independent studies found no interactive effective between EE, contact and clinical outcome (Hogarty, 1985; Dulz \& Hand, 1986). There may be several explanations for this lack of consistency. The most obvious is the difficulty subjects have defining the time spent in 'face-to-face' contact. Other explanations include: confounding effects of clinical state and time spent caring for the patient in cases followed-up at the time of a relapse; and the interaction between the prognostic indicator, poor pre-morbid functioning and high contact between patient and caregiver.

Although it has been stated that contact does not modify the association between low EE and clinical outcome, the relapse rate is doubled $(9 \%$ v. $20 \%$ ) when low contact is maintained with low $E E$ households (Falloon \& McGill, 1985). Further studies may consider the hypothesis that the quality of interpersonal support during aftercare may be associated with clinical outcome. Rather than exploring the association between high $\mathrm{EE}$ and a poor outcome, the protective effects of stable low EE coping behaviours may hold greater clinical significance. The functions that warmth and positive regard expressed towards a patient have in modifying the impact of concurrent criticism have been noted (Brown et al. 1972; Vaughn, 1976). Further study of the benefits of supportive behaviour of caregivers is clearly needed.

Regular ingestion of neuroleptic medication has been another factor considered to modify the predictive power of EE. Again, there are conflicting reports. Vaughn \& Leff $(1976 a)$ showed a significant improvement in the clinical course where medication was ingested in a very regular manner. Vaughn et al (1984) found improvement only when medication was taken regularly by patients who also maintained low contact with high EE household members. Hogarty (1985) found that drug therapy was one of the only two significant determinants of outcome (the other was the number of previous hospital admissions), and that the EE index was not an independent predictor. The patterns of outcome closely paralleled those of placebo-controlled neuroleptic follow-up trials. By contrast the Hamburg study (Dulz \& Hand, 1986) found no benefits for patients taking continuous neuroleptic medication. In naturalistic follow-up studies the precision by which medication ingestion can be defined is limited. Efforts by clinicians to maintain patients on continuous neuroleptic prophylaxis tends to vary according to considerations such as the prior history of the patient and the perceived risk of clinical deterioration. The use of the EE index within the context of a placebo-controlled drug study is probably the only way in which this complex, but crucial interaction can be unravelled.

A recent collaborative study (MacMillan et al. 1986) examined this issue. The findings were similar 
to those of the Hogarty study. The EE index did not independently predict overall outcome, nor was low EE associated with a significantly better response to placebo medication. However, because only one household member was interviewed in most of the cases studied, and the level of critical comments was the only variable used to determine EE status, it is probable that the low EE category may have contained a proportion of households which would have been assigned high EE status in other studies (Vaughn \& Leff, 1986). Thus, the suggestion that patients living in low EE environments may derive limited benefit from continuous neuroleptic prophylaxis remains unanswered. With increasing concern about the dangers associated with long-term continuous neuroleptic treatment (Schooler \& Severe, 1984), the potential of the EE index to target patients who may derive maximal benefits from such therapy deserves further evaluation.

High expressed emotion appears to predict relapse best in single, young males, living in parental households. The predictive value of the EE index is much less impressive in women subjects, who are also more likely to be older and married (Brown et al. 1972; Vaughn \& Leff, 1976a; Hogarty, 1986; Vaughn et al. 1984). Continuous medication and low contact are more prevalent in female cases, but do not fully explain the substantially better outcome for women (Vaughn et al. 1984). The better prognosis of schizophrenia in women, despite similar incidence rates, remains a puzzling phenomenon which the EE construct fails to elucidate.

\section{SPECIFICITY OF EE FOR SCHIZOPHRENIA}

Research linking family environment to aetiology of schizophrenia has found tentative evidence that high EE may precede overt schizophrenia but not affective disorders in behaviourally disturbed adolescents (Goldstein, 1985). The EE index has demonstrated significant predictive; power in determining the prognosis of conditions other than schizophrenia. These have included depressive disorders (Vaughn \& Leff, 1976a; Hooley et al. 1986) and recent onset mania (Miklowitz et al. 1986). In addition, the EE index has been a predictor of adherence to treatment for anorexia nervosa (Szmukler et al. 1985) and the maintenance of weight loss in a weight reduction programme (Fishman-Havstad \& Marston, 1984). Furthermore, the emotional responses of caregivers other than family members have similar predictive value (Brown et al. 1958, 1962).

Thus, the predictive value of the EE index and its components is not restricted to schizophrenic psychopathology and may prove a valuable predictor of the course and management of a wide range of disorders. This raises the intriguing question as to whether the components of the EE index can predict episodes of depression, adherence to treatment, or severe presentations of deficit symptoms in a person with an established diagnosis of schizophrenia.

\section{CONCLUSIONS}

Despite three decades of careful research on the association between household stress factors and the clinical outcome of schizophrenia the precise nature of this association remains obscure. This should not surprise research scientists for whom the precise links between genetic, biochemical and other psychosocial factors and the pathogenesis of schizophrenia have yet to be unravelled. However, the 'expressed emotion' hypothesis has focused attention on the plight of family members who are increasingly expected to cope with the confusing, frightening behaviour of their relatives. Efforts to support the family unit in their efforts to cope with mental disorders are now considered a crucial part of the community management of schizophrenia. Unlike the early efforts that focused on the deficits noted in families under stress, recent developments have attempted to build on the strengths of family coping. The family remains unrivalled in the care of the mentally ill, and is arguably the most valuable resource in promoting the long-term health and welfare of its members. Such an important resource deserves our utmost respect. 


\section{REFERENCES}

Birchwood, M. (1983). Family Coping Behaviour and the Course of Schizophrenia: A Two Year Follow-up Study. Ph.D. thesis, University of Birmingham.

Brown, G W. (1985). The discovery of expressed emotion and its measurement. In Expressed Emotion in Families: Its Significance for Mental Illness (ed. J. Leff and C. Vaughn), pp. 7-25. Guilford Press: New York.

Brown, G. W. \& Rutter, M.(1966). The measurement of family activities and relationships a methodological study Human Relations 19, 241-263.

Brown, G. W., Carstairs, G M. \& Topping, G. (1958). Post-hospital adjustment of chronic mental patients, Lancet ii, 685-689.

Brown, G. W., Monck, E. M., Carstairs, G. M. \& Wing, J. K. (1962) Influence of family life on the course of schizophrenic illness. British Journal of Preventive and Social Medicine 16, 55-68.

Brown, G. W., Birley, J. L. T. \& Wing, J. K. (1972). Influence of family life on the course of schizophrenic disorders: replication. British Journal of Psychiatry 121, 241-258.

Creer, C. \& Wing, J. K. (1974). Schizophrenia at Home. National Schizophrenia Fellowship: London.

Dulz, B. \& Hand, I. (1986) Short-term relapse in young schizophrenics: can it be predicted and affected by family (CFI), patient and treatment variables. In Treatment of Schizophrenta Family Assessment and Intervention (eds M. J. Goldstein, I. Hand and K. Hahlweg), pp. 59-75. Springer-Verlag: Berlin.

Falloon, I. R. H. \& McGill, C. W. (1985). Family stress and the course of schizophrenia: a review. In Family Management of Schizophrenia: A Study of Clmical, Social, Family and Economic Benefits (ed. I. R. H. Falloon), pp. 1-22. Guilford Press: New York.

Falloon, I. R. H., Boyd, J. L., McGill, C. W., Williamson, M., Razani, J., Moss, H. B, Gilderman, A. M. \& Simpson, G. M. (1985). Family management in the prevention of morbidity of schizophrenia: clinical outcome of a two-year longitudinal study. Archives of General Psychiary 42, 887-896.

Fishman-Havstad, L. \& Marston, A. R. (1984). Weight loss maintenance as an aspect of family emotion and process. British Journal of Clinical Psychology 23, 265-271.

Goldstein, M. J. (1985). Family factors that antedate the onset of schizophrenia and related disorders: the results of a fifteen year prospective longitudinal study. Acta Psychiatrica Scandinavica Suppl. No. 319, 71, 7-18.

Grad, J. \& Sainsbury, P. (1963). Mental Illness and the family. Lancet ii, 533-547.

Greenley, J. R. (1979). Family symptom tolerance and rehospitalization experiences of psychiatric patients. Research in Community Menial Health 1, 357-386.

Hatfield, A. B. (1979). The family as partner in the treatment of mental illness. Hospital and Community Psychiatry 30, 338-340.

Hoenig, J. (1974). The schizophrenic patient at home. Acta Psychiatrica Scandinavica 50, 297-308.

Hogarty, G. E. (1985). Expressed emotion and schizophrenic relapse. In Controversies in Schizophrenia (ed. M. Alpert), pp. 354-365, Guilford Press - New York.

Hogarty, G. E., Anderson, C. M., Reiss, D. J., Kornblith, S. J., Greenwald, D. P. Javna, C. D. \& Madonia, M. J. (1986). Family psychoeducation, social skills training and maintenance chemotherapy in the aftercare treatment of schizophrenia: I. One year effects of a controlled study on relapse and expressed emotion. Archives of General Psychiatry 43, 633-642.

Hooley, J. M., Orley, J. \& Teasdale, J. D. (1986). Levels of expressed emotion and relapse in depressed patients. British Journal of Psychiatry 148, 642-647.

Leff, J. \& Vaughn, C. (1980). The interaction of life events and relatives' expressed emotion in schizophrenia and depressive neurosis. British Journal of Psychiatry 136, 146-153.
Leff, J. \& Vaughn, C. (1984). Expressed Emotion in Families: Its Significance for Mental Illness. Guilford Press: New York.

Leff, J. P. \& Kuipers, L., Berkowitz, R., Eberlein-Vries, R. \& Sturgeon, D. (1982). A controlled trial of social intervention in the families of schizophrentc patients British Journal of Psychiatry 141, 121-134.

Leff, J., Kuipers, L., Berkowitz, R., Vaughn, C. \& Sturgeon, D. (1983). Life events, relatives' expressed emotion, and maintenance neuroleptics in schizophrenic relapse. Psychological Medicine 13, 799-807.

MacMillan, J. F., Gold, A., Crow, T. J., Johnson, A. L. \& Johnstone, E. C. (1986). The Northwick Park study of first episodes of schizophrenia. IV. Expressed emotion and relapse. British Journal of Psychiatry 148, 133-143.

Miklowitz, D., Goldstein, M. J. \& Falloon, I. R. H. (1983). Premorbid and symptomatic characteristics of schizophrenics from families with high and low levels of expressed emotion. Journal of Abnormal Psychology 92, 359-367.

Miklowitz, D., Goldstein, M. J., Falloon, I. R. H. \& Doane, J. A. (1984). Interactional characteristics of expressed emotion in the families of schizophrenics. British Journal of Psychiatry 144, 482-487.

Miklowitz, D. J., Goldstein, M. J., Nuechterlein, K. H., Snyder, K. S. \& Doane, J. A. (1986). Expressed emotion, affective style, lithium compliance, and relapse in recent-onset mania Psychopharmacology Bulletin 22, 628-632

Schooler, N. R. \& Severe, J. B. (1984). Efficacy of drug treatment for chronic schizophrenic patients. In The Chronically Mentally III: Research and Services (ed. M. Mirabi), pp. 125-142. Spectrum Publications: New York.

Sturgeon, D., Kuipers, L., Berkowitz, R., Turpin, G. \& Leff, J. P. (1981). Psychophysiological responses of schizophrenic patients to high and low expressed emotion relatives. British Journal of Psychiatry 138, 40-45.

Szmukler, G. I, Eisler, I., Russell, G. F. M. \& Dare, C. (1985). Anorexia nervosa, parental 'expressed emotion' and dropping out of treatment. British Journal of Psychiatry 147, 265-271.

Tarrier, N., Vaughn, C. E., Lader, M. H. \& Leff, J. P. (1979). Bodily reactions to people and events in schizophrenia. Archives of General Psychiatry 36, 311-315.

Test, M. A. \& Stein, L. I. (1980). Alternative to mental hospital treatment. III. Social costs. Archives of General Psychiatry 37, 409-412.

Vaughn, C. E. (1976). The Influence of Family and Social Factors on the Course of Psychiatric Illness: A Comparison of Schizophrenic and Depressed Neurotic Patients. Ph.D. thesis, University of London.

Vaughn, C. (1977). Interaction characteristics in families of schizophrenic patients. In Die andere Seite der Schizophrenie (ed. H. Katschnig), Urban \& Schwarzenberg: Vienna.

Vaughn, C. E. \& Leff, J. P. (1976a). The influence of family and social factors on the course of psychiatric illness: a comparison of schizophrenic and depressed neurotic patients. British Journal of Psychiatry 129, 125-137.

Vaughn, C. E. \& Leff, J. P. (1976b). The measurement of expressed emotion in the families of psychiatric patients. British Journal of Social and Clinical Psychology 15, 157-165.

Vaughn, C. E. \& Leff, J. P. (1981). Patterns of emotional response in relatives of schizophrenic patients. Schizophrenia Bulletin 7, 43-44.

Vaughn, C. \& Leff, J. (1986). First episodes of schizophrenia. British Journal of Psychiatry 148, 215.

Vaughn, C E., Snyder, K. S., Freeman, W., Jones, S. \& Falloon, I. R. H. (1984). Family factors in schizophrenic relapse: a replication in California of the British research on expressed emotion. Archives of General Psychiatry 41, 1169-1177. 